MATHEMATICS OF COMPUTATION

Volume 76, Number 260, October 2007, Pages 1833-1846

S 0025-5718(07)02022-4

Article electronically published on April 30, 2007

\title{
DISCRETE MAXIMUM PRINCIPLE FOR HIGHER-ORDER FINITE ELEMENTS IN 1D
}

\author{
TOMÁŠ VEJCHODSKÝ AND PAVEL ŠOLÍN
}

\begin{abstract}
We formulate a sufficient condition on the mesh under which we prove the discrete maximum principle (DMP) for the one-dimensional Poisson equation with Dirichlet boundary conditions discretized by the $h p$-FEM. The DMP holds if a relative length of every element $K$ in the mesh is bounded by a value $H_{\text {rel }}^{*}(p) \in[0.9,1]$, where $p \geq 1$ is the polynomial degree of the element $K$. The values $H_{\text {rel }}^{*}(p)$ are calculated for $1 \leq p \leq 100$.
\end{abstract}

\section{INTRODUCTION}

Classical (continuous) maximum principles belong to the most important results in the theory of second-order partial differential equations (PDEs). Their discrete counterparts, discrete maximum principles (DMP), appeared in the early 1970s. They were used by various authors to prove the convergence of the lowest-order finite difference and finite element methods (see, e.g., [3, 4] and the references therein). DMP have been studied intensively during the past decades in the context of linear PDEs [2, 8, 10, 17, 18, 20, and more recently also nonlinear equations 9]. Most of these results have two points in common:

- they are limited to lowest-order approximations,

- they are based on $M$-matrices [6, 16].

Much less is known about the DMP for methods of higher orders of accuracy such as higher-order finite difference methods, spectral FEM, or $h p$-FEM. Let us mention, e.g., a result [21] on higher-order collocation methods. Particularly noteworthy is a negative result [7] from 1981 stating that a stronger DMP is not valid for cubic and higher-order Lagrange elements in 2D. In the quadratic case, the stronger DMP is valid under extremely restrictive assumptions on the mesh, which almost never could be satisfied in practice. In light of this negative result, a few attempts were made to formulate and prove weakened forms of the DMP (see, e.g., 11, 14]). The present result is based on the analysis of the discrete Green's function (DGF) for higher-order elements. A similar concept was used in the piecewise-linear case in [5].

The paper is organized as follows. In Section 2 we introduce the one-dimensional Poisson problem, its $h p$-FEM discretization, and the discrete maximum principle.

Received by the editor January 31, 2006 and, in revised form, July 25, 2006.

2000 Mathematics Subject Classification. Primary 65N30; Secondary 35B50.

Key words and phrases. Discrete maximum principle, discrete Green's function, higher-order elements, $h p$-FEM, Poisson equation.

(C)2007 American Mathematical Society Reverts to public domain 28 years from publication 1833 
The discrete Green's function along with its basic properties is discussed in Section 3 . In Section 4 we derive an explicit formula for the DGF for the Poisson problem discretized by $h p$-FEM, which is used to find sufficient conditions for its nonnegativity in Section 5 This leads to the notion of critical relative element length $H_{\mathrm{rel}}^{*}$. The main result is presented in Section 6 .

\section{Model problem AND its Discretization}

We consider the one-dimensional Poisson equation with homogeneous Dirichlet boundary conditions in an open bounded interval $\Omega=(\alpha, \beta)$. The standard weak formulation reads: Find $u \in V=H_{0}^{1}(\Omega)$ such that

$$
a(u, v)=(f, v) \quad \forall v \in V
$$

where $f \in L^{2}(\Omega)$, the symbol $(\cdot, \cdot)$ stands for the inner product in $L^{2}(\Omega), H_{0}^{1}(\Omega)$ is the standard Sobolev space, and $a(u, v)=\left(u^{\prime}, v^{\prime}\right)$.

We create a partition $\alpha=x_{0}<x_{1}<\ldots<x_{M}=\beta$ of the domain $\Omega$ consisting of $M$ elements $K_{i}=\left[x_{i-1}, x_{i}\right], i=1,2, \ldots, M$. Every element $K_{i}$ is assigned an arbitrary polynomial degree $p_{i} \geq 1$. The corresponding finite element space of piecewise-polynomial continuous functions $V_{h p} \subset V$ has the form

$$
V_{h p}=\left\{v_{h p} \in V ;\left.v_{h p}\right|_{K_{i}} \in P^{p_{i}}\left(K_{i}\right), i=1,2, \ldots, M\right\},
$$

where $P^{p_{i}}\left(K_{i}\right)$ stands for the space of polynomials of degree at most $p_{i}$ on the element $K_{i}$. The space $V_{h p}$ has the dimension $N=-1+\sum_{i=1}^{M} p_{i}$. There exists a unique function $u_{h p} \in V_{h p}$ satisfying

$$
a\left(u_{h p}, v_{h p}\right)=\left(f, v_{h p}\right) \quad \forall v_{h p} \in V_{h p} .
$$

Definition 2.1. We say that problem (2.2) satisfies the discrete maximum principle (DMP) if for any right-hand side $f \in L^{2}(\Omega)$ it holds that

$$
f \geq 0 \text { a.e. in } \Omega \Rightarrow u_{h p} \geq 0 \text { in } \Omega \text {. }
$$

Remark 2.2. The above implication is equivalent to

$$
f \geq 0 \text { a.e. in } \Omega \Rightarrow \min _{x \in \bar{\Omega}} u_{h p}(x)=\min _{x \in \partial \Omega} u_{h p}(x)
$$

for homogeneous Dirichlet boundary conditions. This is further equivalent to

$$
f \leq 0 \text { a.e. in } \Omega \Rightarrow \max _{x \in \bar{\Omega}} u_{h p}(x)=\max _{x \in \partial \Omega} u_{h p}(x) .
$$

Remark 2.3. In problem (2.2), homogeneous Dirichlet conditions are considered without loss of generality. This follows immediately from the fact that every solution $\hat{u}_{h p}$ to a problem with nonhomogeneous Dirichlet boundary conditions can be written as $\hat{u}_{h p}=u_{h p}^{L}+u_{h p}$, where $u_{h p}^{L}$ is a linear function satisfying the nonhomogeneous conditions and $u_{h p}$ vanishes at $\Omega$-endpoints.

\section{Discrete Green's function}

The discrete Green's function (DGF) is defined in analogy with the standard (continuous) Green's function:

Definition 3.1. For an arbitrary $z \in \Omega$, the unique solution $G_{h p, z} \in V_{h p}$ to the problem

$$
a\left(v_{h p}, G_{h p, z}\right)=v_{h p}(z) \quad \forall v_{h p} \in V_{h p}
$$

is called the discrete Green's function (DGF) corresponding to the point $z$. 
In the following, we will use the notation $G_{h p}(x, z)=G_{h p, z}(x)$. A combination of (2.2) and (3.1) yields an important consequence:

$$
u_{h p}(z)=\int_{\Omega} G_{h p}(x, z) f(x) \mathrm{d} x \quad \forall z \in \Omega .
$$

The following lemma shows that the DGF can easily be expressed using any basis of $V_{h p}$; cf. [5]. We use the Kronecker symbol

$$
\delta_{i k}= \begin{cases}1 & \text { for } i=k \\ 0 & \text { for } i \neq k\end{cases}
$$

Lemma 3.2. Let $\left\{\varphi_{1}, \varphi_{2}, \ldots, \varphi_{N}\right\}$ be any basis of $V_{h p}$. If the stiffness matrix $A_{i j}=a\left(\varphi_{j}, \varphi_{i}\right), 1 \leq i, j \leq N$, is nonsingular, then

$$
G_{h p}(x, z)=\sum_{j=1}^{N} \sum_{k=1}^{N} A_{j k}^{-1} \varphi_{k}(x) \varphi_{j}(z)
$$

Here $A_{j k}^{-1}$ are the entries of the inverse stiffness matrix, i.e., $\sum_{j=1}^{N} A_{i j} A_{j k}^{-1}=\delta_{i k}$, $1 \leq i, k \leq N$

Proof. Substitute

$$
G_{h p}(x, z)=\sum_{i=1}^{N} c_{i}(z) \varphi_{i}(x)
$$

into (3.1) with $v_{h p}=\varphi_{j}$. It follows that

$$
\sum_{i=1}^{N} c_{i}(z) \underbrace{a\left(\varphi_{j}, \varphi_{i}\right)}_{A_{i j}}=\varphi_{j}(z) .
$$

The coefficients $c_{i}(z)$ can be expressed in terms of the inverse matrix as $c_{k}(z)=$ $\sum_{j=1}^{N} \varphi_{j}(z) A_{j k}^{-1}$, and they can be substituted back into (3.4).

Corollary 3.3. Let $\left\{l_{1}, l_{2}, \cdots, l_{N}\right\}$ be a basis of $V_{h p}$ such that $a\left(l_{i}, l_{j}\right)=\delta_{i j}$. Then

$$
G_{h p}(x, z)=\sum_{i=1}^{N} l_{i}(x) l_{i}(z) .
$$

Lemma 3.4. If there exists a basis $\left\{l_{1}, l_{2}, \ldots, l_{N}\right\}$ of $V_{h p}$ such that $a\left(l_{i}, l_{j}\right)=\delta_{i j}$, $1 \leq i, j \leq N$, then $G_{h p}(x, x)>0$ for all $x \in \Omega$.

Proof. Let $x \in \Omega$. Since $\left\{l_{1}, l_{2}, \ldots, l_{N}\right\}$ is a basis, there exists at least one $k \in$ $\{1,2, \ldots, N\}$ such that $l_{k}(x) \neq 0$. Hence, by Corollary 3.3

$$
G_{h p}(x, x)=\sum_{i=1}^{N} l_{i}^{2}(x)>0 .
$$

Theorem 3.5. Problem (2.2) satisfies the discrete maximum principle if and only if the corresponding discrete Green's function $G_{h p}(x, z)=G_{h p, z}(x)$ defined by (3.1) is nonnegative in $\Omega^{2}$.

Proof. Immediate consequence of (3.2). 
Remark 3.6. Results presented in this section are valid for any second-order elliptic problem of the form (2.1) as well as in higher spatial dimensions.

\section{DGF FOR Poisson PROBlem IN 1D}

4.1. Lowest-order case. Consider the case $p_{1}=p_{2}=\ldots=p_{M}=1$ first. Let $\mathcal{B}^{L}=\left\{\phi_{1}, \phi_{2}, \ldots, \phi_{M-1}\right\}$ be the standard lowest-order basis consisting of the piecewise-linear "hat functions" such that $\phi_{j}\left(x_{i}\right)=\delta_{i j}, 1 \leq i, j \leq M-1$. In this case the stiffness matrix $A^{L} \in \mathbb{R}^{(M-1) \times(M-1)}$ is tridiagonal,

$$
A_{i j}^{L}= \begin{cases}1 / h_{i}+1 / h_{i+1} & \text { for } i=j, \\ -1 / h_{i+1} & \text { for } i=j-1, \\ -1 / h_{i-1} & \text { for } i=j+1, \\ 0 & \text { otherwise }\end{cases}
$$

with $h_{i}=x_{i}-x_{i-1}$.

Lemma 4.1. The inverse matrix $\left(A^{L}\right)^{-1} \in \mathbb{R}^{(M-1) \times(M-1)}$ has the form

$$
\left(A^{L}\right)^{-1}=\frac{1}{\beta-\alpha}\left(\begin{array}{cccc}
\left(x_{1}-\alpha\right)\left(\beta-x_{1}\right) & \left(x_{1}-\alpha\right)\left(\beta-x_{2}\right) & \left(x_{1}-\alpha\right)\left(\beta-x_{3}\right) & \ldots \\
\left(x_{1}-\alpha\right)\left(\beta-x_{2}\right) & \left(x_{2}-\alpha\right)\left(\beta-x_{2}\right) & \left(x_{2}-\alpha\right)\left(\beta-x_{3}\right) & \ldots \\
\left(x_{1}-\alpha\right)\left(\beta-x_{3}\right) & \left(x_{2}-\alpha\right)\left(\beta-x_{3}\right) & \left(x_{3}-\alpha\right)\left(\beta-x_{3}\right) & \ldots \\
\vdots & \vdots & \vdots & \ddots
\end{array}\right),
$$

i.e., $\left(A^{L}\right)_{i j}^{-1}=\left(x_{i}-\alpha\right)\left(\beta-x_{j}\right) /(\beta-\alpha)$ for $1 \leq i \leq j \leq M-1$ and $\left(A^{L}\right)_{i j}^{-1}=$ $\left(x_{j}-\alpha\right)\left(\beta-x_{i}\right) /(\beta-\alpha)$ for $1 \leq j<i \leq M-1$.

Proof 1 . We need to show that $z_{i j}=\delta_{i j}$, where

$$
z_{i j}=\sum_{k=1}^{M-1}\left(A^{L}\right)_{i k}^{-1} A_{k j}^{L}=\sum_{k=1}^{M-1}\left(A^{L}\right)_{i k}^{-1} a\left(\phi_{j}, \phi_{k}\right),
$$

for all $i, j=1,2, \ldots, M-1$. Let us fix $i$ and $j$ and consider the bilinear forms

$$
a_{1}(u, v)=\int_{\alpha}^{x_{i}} u^{\prime} v^{\prime} \mathrm{d} x \quad \text { and } \quad a_{2}(u, v)=\int_{x_{i}}^{\beta} u^{\prime} v^{\prime} \mathrm{d} x .
$$

The explicit formulae for $\left(A^{L}\right)_{i k}^{-1}$ yield

$$
\begin{aligned}
(\beta-\alpha) z_{i j}=\left(\beta-x_{i}\right) a\left(\phi_{j}, \sum_{k=1}^{i-1}\left(x_{k}-\alpha\right) \phi_{k}\right) & +\left(x_{i}-\alpha\right)\left(\beta-x_{i}\right) a\left(\phi_{j}, \phi_{i}\right) \\
& +\left(x_{i}-\alpha\right) a\left(\phi_{j}, \sum_{k=i+1}^{M-1}\left(\beta-x_{k}\right) \phi_{k}\right) .
\end{aligned}
$$

Now, we split the term $a\left(\phi_{j}, \phi_{i}\right)=a_{1}\left(\phi_{j}, \phi_{i}\right)+a_{2}\left(\phi_{j}, \phi_{i}\right)$ to obtain

$$
(\beta-\alpha) z_{i j}=\left(\beta-x_{i}\right) a_{1}\left(\phi_{j}, x-\alpha\right)+\left(x_{i}-\alpha\right) a_{2}\left(\phi_{j}, \beta-x\right) .
$$

The fact that $a_{1}\left(\phi_{j}, \beta-x\right)=a_{2}\left(\phi_{j}, x-\alpha\right)=\delta_{i j}$ finishes the proof.

\footnotetext{
${ }^{1}$ The authors thank an anonymous referee for simplifying their original proof.
} 


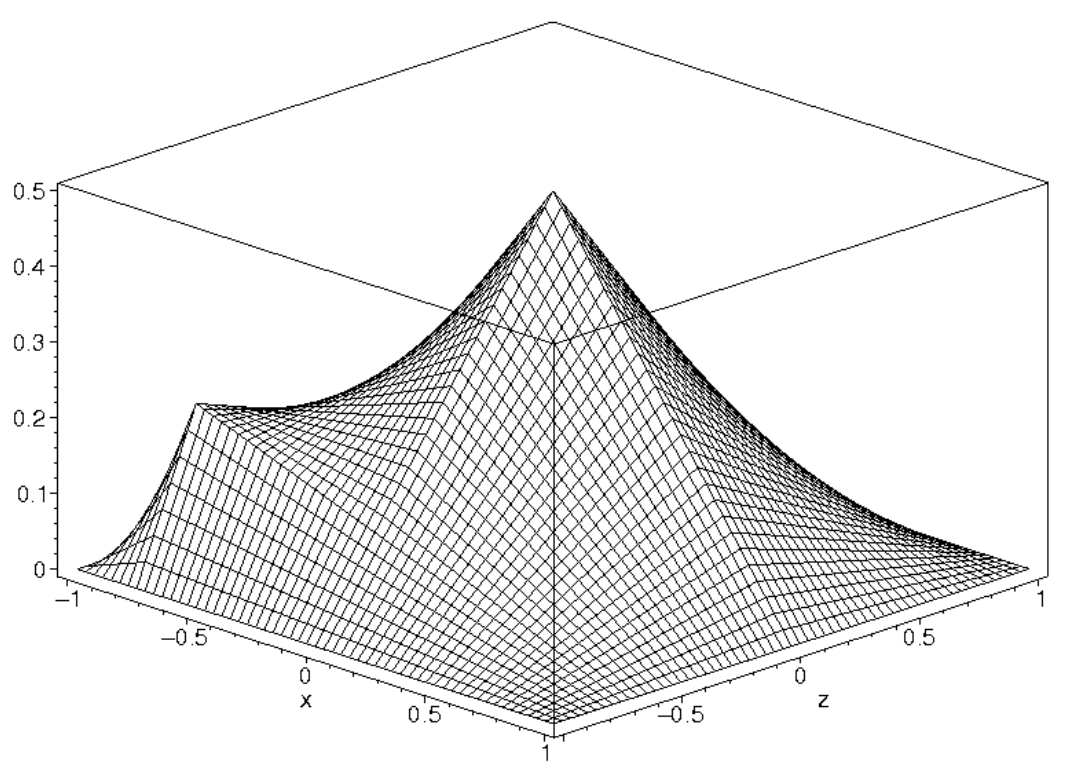

FIgURE 1. The lowest-order part $G_{h p}^{L}(x, z)$ of the discrete Green's function $G_{h p}(x, z)$ for the Poisson equation in $\Omega=(-1,1)$, on a mesh with three elements $[-1,-3 / 4],[-3 / 4,0]$, and $[0,1]$.

Using Lemma 4.1 and identity (3.3), we can write the DGF in the form

$$
\begin{aligned}
G_{h p}^{L}(x, z)= & \frac{1}{\beta-\alpha}\left(\sum_{i=1}^{M-1}\left(x_{i}-\alpha\right)\left(\beta-x_{i}\right) \phi_{i}(x) \phi_{i}(z)\right. \\
& \left.+\sum_{i=1}^{M-2} \sum_{j=i+1}^{M-1}\left(x_{i}-\alpha\right)\left(\beta-x_{j}\right)\left[\phi_{i}(x) \phi_{j}(z)+\phi_{j}(x) \phi_{i}(z)\right]\right) .
\end{aligned}
$$

In particular, we see immediately that

$$
G_{h p}^{L}(x, z) \geq 0 \quad \forall[x, z] \in \Omega^{2} .
$$

The situation is illustrated in Figure 1

4.2. Higher-order case. In this paragraph we return to the original setting with arbitrary polynomial degrees $p_{i} \geq 1$. In order to facilitate the construction of higher-order basis functions of the space $V_{h p}$, let us introduce the Lobatto shape functions $l_{0}, l_{1}, l_{2}, \ldots$ on a reference interval $\hat{K}=[-1,1]$ (see, e.g., [12, 15]).

The lowest-order Lobatto shape functions $l_{0}$ and $l_{1}$ have the form $l_{0}(\xi)=$ $(1-\xi) / 2, l_{1}(\xi)=(1+\xi) / 2, \xi \in \hat{K}$. The higher-order shape functions $l_{2}, l_{3}, \ldots$ are defined as antiderivatives to the Legendre polynomials. Therefore, they satisfy

$$
\int_{-1}^{1} l_{i}^{\prime}(\xi) l_{j}^{\prime}(\xi) \mathrm{d} \xi=\delta_{i j}, \quad i, j=2,3, \ldots
$$


Every Lobatto shape function $l_{i}, i=2,3, \ldots$, is a polynomial of degree $i$ and it vanishes at \pm 1 . Thus it can be expressed as

$$
l_{i}(\xi)=l_{0}(\xi) l_{1}(\xi) \kappa_{i}(\xi), \quad i=2,3, \ldots,
$$

where $\kappa_{i}$ is a polynomial of degree $i-2$. For reference, the first few kernels $\kappa_{i}$ are listed in Appendix.

The basis $\mathcal{B}=\left\{\phi_{1}, \phi_{2}, \ldots, \phi_{N}\right\}$ of $V_{h p}$ can be written as $\mathcal{B}=\mathcal{B}^{L} \cup \mathcal{B}^{B}$, where $\mathcal{B}^{L}$ was defined above and $\mathcal{B}^{B}$ is the higher-order part of the basis comprising functions $\phi_{M}, \phi_{M+1}, \ldots, \phi_{N}$. These are defined as follows.

Consider the standard linear transformations from $\hat{K}$ to $K_{i}$,

$$
\chi_{K_{i}}(\xi)=\frac{\left(x_{i}-x_{i-1}\right) \xi+\left(x_{i}+x_{i-1}\right)}{2} .
$$

On an element $K_{i}$ of the polynomial degree $p_{i}$, there are $p_{i}-1$ higher-order basis functions. These vanish outside of $K_{i}$ and in $K_{i}$ they are defined as the Lobatto shape functions $l_{2}, l_{3}, \ldots, l_{p_{i}}$ composed with the inverse map $\chi_{K_{i}}^{-1}(x)$.

Proposition 4.2. We have the following orthogonality relations:

$$
\begin{array}{ll}
a\left(\phi^{L}, \phi^{B}\right)=0 & \forall \phi^{L} \in \mathcal{B}^{L}, \forall \phi^{B} \in \mathcal{B}^{B}, \\
a\left(\phi^{B}, \psi^{B}\right)=0 & \forall \phi^{B} \in \mathcal{B}^{B}, \forall \psi^{B} \in \mathcal{B}^{B}, \phi^{B} \neq \psi^{B} .
\end{array}
$$

Proof. The proof is straightforward, based on the $L^{2}$-orthogonality of the Legendre polynomials.

By Proposition 4.2, both the stiffness matrix $A$ and its inverse have the following block structure:

$$
A=\left(\begin{array}{cc}
A^{L} & 0 \\
0 & D
\end{array}\right), \quad A^{-1}=\left(\begin{array}{cc}
\left(A^{L}\right)^{-1} & 0 \\
0 & D^{-1}
\end{array}\right)
$$

with

$$
D=\operatorname{diag}(\underbrace{\frac{2}{h_{1}}, \ldots, \frac{2}{h_{1}}}_{\left(p_{1}-1\right) \text { times }}, \underbrace{\frac{2}{h_{2}}, \ldots, \frac{2}{h_{2}}}_{\left(p_{2}-1\right) \text { times }}, \ldots, \underbrace{\left.\frac{2}{h_{M}}, \ldots, \frac{2}{h_{M}}\right)}_{\left(p_{M}-1\right) \text { times }} .
$$

By (3.3), the DGF can be written as

$$
G_{h p}(x, z)=G_{h p}^{L}(x, z)+G_{h p}^{B}(x, z),
$$

where $G_{h p}^{L}(x, z)$ corresponds to (4.1) and

$$
G_{h p}^{B}(x, z)=\sum_{k=M}^{N} D_{k k}^{-1} \phi_{k}(x) \phi_{k}(z) \quad \forall[x, z] \in \Omega^{2} .
$$

Unfortunately, $G_{h p}^{B}(x, z)$ defined by (4.6) is not nonnegative in the entire $\Omega^{2}$ in general. For instance, in the example shown in Figure 2, there are small regions near the points $[1,0]$ and $[0,1]$, where the function $G_{h p}^{B}(x, z)$ is negative.

Notice that any partition of $\Omega$ produces a rectangular grid on $\Omega^{2}$ and that $G_{h p}^{B}(x, z)$ can be nonzero within the diagonal squares of this grid only. In other words,

$$
\operatorname{supp} G_{h p}^{B} \subset \bigcup_{i=1}^{M} K_{i}^{2}
$$




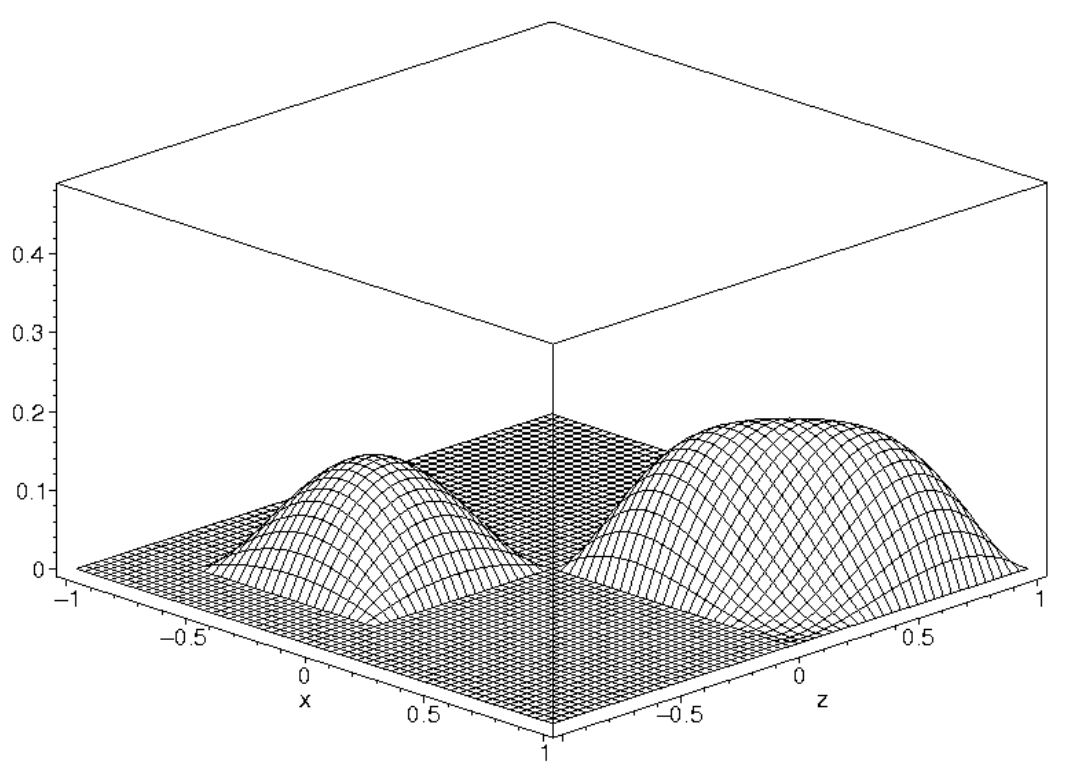

FIGURE 2. The higher-order part $G_{h p}^{B}(x, z)$ of the discrete Green's function $G_{h p}(x, z)$ for the Poisson equation in $\Omega=(-1,1)$, on a mesh with three elements $[-1,-3 / 4],[-3 / 4,0]$, and $[0,1]$ of the polynomial degrees $p_{1}=1, p_{2}=2, p_{3}=3$.

Lemma 4.3. The discrete Green's function $G_{h p}$ defined by (4.5) is nonnegative in $\Omega^{2} \backslash \bigcup_{i=1}^{M} K_{i}^{2}$.

Proof. Consider (4.7) together with (4.2).

\section{The DGF ON $K_{i}^{2}$}

As justified by Lemma 4.3 , we only need to continue with the study of the discrete Green's function $G_{h p}(x, z)$ in the union of the diagonal squares $\bigcup_{i=1}^{M} K_{i}^{2}$. Without loss of generality, let us restrict ourselves to only one square $K_{i}^{2}, 1 \leq i \leq M$. Let $p=p_{i}$ be the polynomial degree assigned to $K_{i}$. Notice that only a few terms in (4.1) and (4.6) are nonzero in $K_{i}^{2}$. Hence, by (4.1), (4.4), and (4.6) we obtain

$$
\begin{aligned}
\left.G_{h p}(x, z)\right|_{K_{i}^{2}}= & \frac{\left(x_{i}-\alpha\right)\left(\beta-x_{i}\right)}{\beta-\alpha} \phi_{i}(x) \phi_{i}(z) \\
& +\frac{\left(x_{i-1}-\alpha\right)\left(\beta-x_{i-1}\right)}{\beta-\alpha} \phi_{i-1}(x) \phi_{i-1}(z) \\
& +\frac{\left(x_{i-1}-\alpha\right)\left(\beta-x_{i}\right)}{\beta-\alpha}\left[\phi_{i}(x) \phi_{i-1}(z)+\phi_{i-1}(x) \phi_{i}(z)\right] \\
& +\frac{x_{i}-x_{i-1}}{2} G_{h p}^{B}(x, z),
\end{aligned}
$$

$[x, z] \in K_{i}^{2}, 1 \leq i \leq M$. It is convenient to introduce the notation $K_{i}=\left[x_{i-1}, x_{i}\right]=$ $[L, R]$. 
We transform the function $G_{h p}$ from $K_{i}^{2}$ to the reference square $\hat{K}^{2}=[-1,1]^{2}$ using the linear transformation (4.3) with $x=\chi_{K_{i}}(\xi)$ and $z=\chi_{K_{i}}(\eta)$,

$$
\begin{aligned}
\left.G_{h p}(x, z)\right|_{K_{i}^{2}}=\hat{G}_{h p}(\xi, \eta)= & \frac{(R-\alpha)(\beta-R)}{\beta-\alpha} l_{1}(\xi) l_{1}(\eta) \\
& +\frac{(L-\alpha)(\beta-L)}{\beta-\alpha} l_{0}(\xi) l_{0}(\eta) \\
& +\frac{(L-\alpha)(\beta-R)}{\beta-\alpha}\left[l_{1}(\xi) l_{0}(\eta)+l_{0}(\xi) l_{1}(\eta)\right] \\
& +\frac{R-L}{2} \hat{G}_{h p}^{p, B}(\xi, \eta),
\end{aligned}
$$

$[\xi, \eta] \in \hat{K}^{2}$. Here $l_{0}(\xi)$ and $l_{1}(\xi)$ are the above-defined lowest-order shape functions on $\hat{K}$ and

$$
\hat{G}_{h p}^{p, B}(\xi, \eta)=\sum_{k=2}^{p} l_{k}(\xi) l_{k}(\eta)=l_{0}(\xi) l_{0}(\eta) l_{1}(\xi) l_{1}(\eta) \sum_{k=2}^{p} \kappa_{k}(\xi) \kappa_{k}(\eta)
$$

is the higher-order part.

Let us modify formula (5.2) in the following way: Divide (5.2) by $R-L>0$ and use the identities

$$
\begin{aligned}
& \frac{(L-\alpha)(\beta-L)}{(\beta-\alpha)(R-L)}=\frac{(L-\alpha)(\beta-R)}{(\beta-\alpha)(R-L)}+\frac{L-\alpha}{\beta-\alpha}, \\
& \frac{(R-\alpha)(\beta-R)}{(\beta-\alpha)(R-L)}=\frac{(L-\alpha)(\beta-R)}{(\beta-\alpha)(R-L)}+\frac{\beta-R}{\beta-\alpha},
\end{aligned}
$$

and

$$
l_{0}(\xi) l_{0}(\eta)+l_{1}(\xi) l_{1}(\eta)+l_{0}(\xi) l_{1}(\eta)+l_{1}(\xi) l_{0}(\eta)=1 \quad \forall[\xi, \eta] \in \hat{K}^{2} .
$$

We obtain

$$
\begin{aligned}
\frac{\hat{G}_{h p}(\xi, \eta)}{R-L}= & \frac{(L-\alpha)(\beta-R)}{(\beta-\alpha)(R-L)}+\frac{L-\alpha}{\beta-\alpha} l_{0}(\xi) l_{0}(\eta) \\
& +\frac{\beta-R}{\beta-\alpha} l_{1}(\xi) l_{1}(\eta)+\frac{1}{2} \hat{G}_{h p}^{p, B}(\xi, \eta) .
\end{aligned}
$$

The endpoints of $K_{i}$ can be parameterized using the element length $H=R-L$ and a real parameter $0 \leq t \leq 1$, so that $L=\alpha$ for $t=0$ and $R=\beta$ for $t=1$ :

$$
\begin{aligned}
& L=(1-t) \alpha+t(\beta-H), \\
& R=(1-t)(\alpha+H)+t \beta .
\end{aligned}
$$

Use (5.5) and (5.6), define relative element length $H_{\text {rel }}$ by

$$
H_{\mathrm{rel}}=\frac{H}{\beta-\alpha},
$$

and compute

$$
\begin{aligned}
\frac{L-\alpha}{\beta-\alpha} & =\frac{t(\beta-\alpha-H)}{\beta-\alpha}=t\left(1-H_{\text {rel }}\right), \\
\frac{\beta-R}{\beta-\alpha} & =\frac{(1-t)(\beta-\alpha-H)}{\beta-\alpha}=(1-t)\left(1-H_{\text {rel }}\right), \\
\frac{(L-\alpha)(\beta-R)}{(\beta-\alpha)(R-L)} & =\frac{t(1-t)(\beta-\alpha-H)^{2}}{(\beta-\alpha) H}=t(1-t) \frac{\left(1-H_{\text {rel }}\right)^{2}}{H_{\text {rel }}} .
\end{aligned}
$$


Substitute (5.7)-(5.9) into (5.4) to obtain

$$
\begin{aligned}
& \frac{\hat{G}_{h p}(\xi, \eta)}{H}=t(1-t) \frac{\left(1-H_{\mathrm{rel}}\right)^{2}}{H_{\mathrm{rel}}}+t\left(1-H_{\mathrm{rel}}\right) l_{0}(\xi) l_{0}(\eta) \\
& \quad+(1-t)\left(1-H_{\mathrm{rel}}\right) l_{1}(\xi) l_{1}(\eta)+\frac{1}{2} \hat{G}_{h p}^{p, B}(\xi, \eta) .
\end{aligned}
$$

Finally, use the identity

$$
\hat{G}_{h p}^{p, B}(\xi, \eta)=t \hat{G}_{h p}^{p, B}(\xi, \eta)+(1-t) \hat{G}_{h p}^{p, B}(\xi, \eta)
$$

substitute (5.3) into (5.10), and factor out $l_{0}(\xi) l_{0}(\eta)$ and $l_{1}(\xi) l_{1}(\eta)$ :

$$
\begin{aligned}
\frac{\hat{G}_{h p}(\xi, \eta)}{H}= & t(1-t) \frac{\left(1-H_{\text {rel }}\right)^{2}}{H_{\text {rel }}} \\
& +t l_{0}(\xi) l_{0}(\eta)\left[1-H_{\text {rel }}+\frac{1}{2} l_{1}(\xi) l_{1}(\eta) \sum_{k=2}^{p} \kappa_{k}(\xi) \kappa_{k}(\eta)\right] \\
& +(1-t) l_{1}(\xi) l_{1}(\eta)\left[1-H_{\text {rel }}+\frac{1}{2} l_{0}(\xi) l_{0}(\eta) \sum_{k=2}^{p} \kappa_{k}(\xi) \kappa_{k}(\eta)\right] .
\end{aligned}
$$

Indeed, the value $t(1-t)\left(1-H_{\text {rel }}\right)^{2} / H_{\text {rel }}$ is nonnegative for all $t \in[0,1]$ as well as the values $t l_{0}(\xi) l_{0}(\eta)$ and $(1-t) l_{1}(\xi) l_{1}(\eta)$, for all $[\xi, \eta] \in \hat{K}^{2}$. Hence, the discrete Green's function $G_{h p}$ is nonnegative in $K_{i}^{2}$ if both expressions in the square brackets in (5.11) are nonnegative. To see that they impose the same restriction on the relative element length $H_{\text {rel }}$, let us introduce Lemma 5.1;

Lemma 5.1. It is true that

$$
\min _{[\xi, \eta] \in \hat{K}^{2}} l_{0}(\xi) l_{0}(\eta) \sum_{k=2}^{p} \kappa_{k}(\xi) \kappa_{k}(\eta)=\min _{[\xi, \eta] \in \hat{K}^{2}} l_{1}(\xi) l_{1}(\eta) \sum_{k=2}^{p} \kappa_{k}(\xi) \kappa_{k}(\eta) .
$$

Proof. Using the definition of the functions $\kappa_{i}$, it is easy to see that $\kappa_{k}(\xi)=\kappa_{k}(-\xi)$ for $k$ even and $\kappa_{k}(\xi)=-\kappa_{k}(-\xi)$ for $k$ odd. Therefore, $\kappa_{k}(\xi) \kappa_{k}(\eta)=\kappa_{k}(-\xi) \kappa_{k}(-\eta)$ for every $k=2,3, \ldots$ Moreover, $l_{0}(\xi)=l_{1}(-\xi)$, which yields

$$
\begin{aligned}
\min _{[\xi, \eta] \in \hat{K}^{2}} l_{0}(\xi) l_{0}(\eta) \sum_{k=2}^{p} \kappa_{k}(\xi) \kappa_{k}(\eta) & =\min _{[\xi, \eta] \in \hat{K}^{2}} l_{1}(-\xi) l_{1}(-\eta) \sum_{k=2}^{p} \kappa_{k}(-\xi) \kappa_{k}(-\eta) \\
& =\min _{[\xi, \eta] \in \hat{K}^{2}} l_{1}(\xi) l_{1}(\eta) \sum_{k=2}^{p} \kappa_{k}(\xi) \kappa_{k}(\eta) .
\end{aligned}
$$

Relation (5.11) and Lemma 5.1 motivate the following definition:

Definition 5.2. By critical relative element length $H_{\text {rel }}^{*}$ corresponding to a polynomial degree $p \geq 2$ we mean the value

$$
\begin{aligned}
H_{\mathrm{rel}}^{*}(p) & =1+\frac{1}{2} \min _{(\xi, \eta) \in \hat{K}^{2}} l_{0}(\xi) l_{0}(\eta) \sum_{k=2}^{p} \kappa_{k}(\xi) \kappa_{k}(\eta) \\
& =1+\frac{1}{2} \min _{(\xi, \eta) \in \hat{K}^{2}} l_{1}(\xi) l_{1}(\eta) \sum_{k=2}^{p} \kappa_{k}(\xi) \kappa_{k}(\eta) .
\end{aligned}
$$

For $p=1$ we define $H_{\text {rel }}^{*}=1$. 


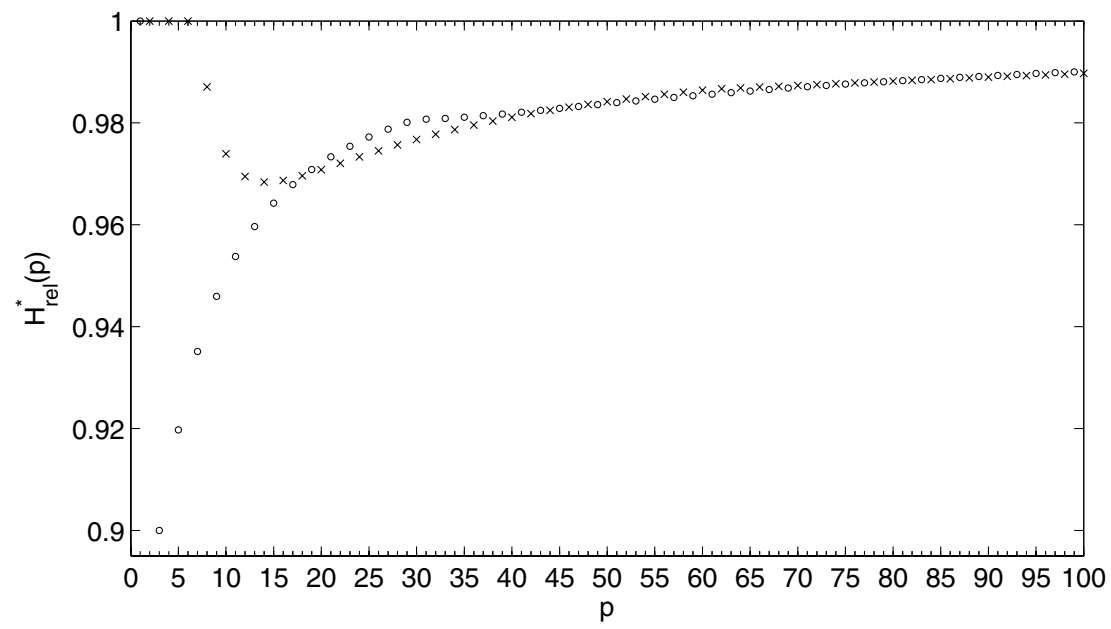

Figure 3. Critical relative element lengths $H_{\text {rel }}^{*}(p)$ for $p=$ $1,2, \ldots, 100$. Circles indicate the values for $p$ odd and crosses indicate the value for $p$ even.

Theorem 5.3. If $\alpha \leq L<R \leq \beta$ and

$$
\frac{R-L}{\beta-\alpha} \leq H_{\mathrm{rel}}^{*}(p)
$$

then the function $\hat{G}_{h p}(\xi, \eta)$ defined by (5.2) is nonnegative for all $[\xi, \eta] \in \hat{K}^{2}=$ $[-1,1]^{2}$.

Proof. Apply (5.13) and the definition of $H_{\text {rel }}^{*}(p)$ to infer

$$
\begin{aligned}
1-H_{\mathrm{rel}}+\frac{1}{2} l_{1}(\xi) & l_{1}(\eta) \sum_{k=2}^{p} \kappa_{k}(\xi) \kappa_{k}(\eta) \\
& \geq 1-H_{\mathrm{rel}}^{*}(p)+\frac{1}{2} l_{1}(\xi) l_{1}(\eta) \sum_{k=2}^{p} \kappa_{k}(\xi) \kappa_{k}(\eta) \geq 0 \quad \forall[\xi, \eta] \in \hat{K}^{2} .
\end{aligned}
$$

Similarly,

$$
1-H_{\mathrm{rel}}+\frac{1}{2} l_{0}(\xi) l_{0}(\eta) \sum_{k=2}^{p} \kappa_{k}(\xi) \kappa_{k}(\eta) \geq 0 \quad \forall[\xi, \eta] \in \hat{K}^{2} .
$$

Thus, all terms in (5.11) are nonnegative and we can conclude that

$$
\hat{G}_{h p}(\xi, \eta) \geq 0 \text { for all }[\xi, \eta] \in \hat{K}^{2} .
$$

Computation of $H_{\mathrm{rel}}^{*}(p)$. In Table 1 we list the values of $H_{\text {rel }}^{*}(p)$ for $p=1,2, \ldots, 20$.

The values of $H_{\text {rel }}^{*}(p)$ for $p=1,2, \ldots, 100$ are plotted in Figure 3. While the values $H_{\text {rel }}^{*}(p)$ for $p=1,2,3,4$ could be calculated analytically, results for $p \geq 5$ are numerical, obtained with high accuracy. 
TABLE 1. Critical relative element length $H_{\text {rel }}^{*}(p)$ for $p=1,2,3, \ldots, 20$.

\begin{tabular}{cc}
\hline$p$ & $H_{\mathrm{rel}}^{*}(p)$ \\
\hline 1 & 1 \\
2 & 1 \\
3 & $9 / 10$ \\
4 & 1 \\
5 & 0.919731 \\
\hline
\end{tabular}

\begin{tabular}{cc}
\hline$p$ & $H_{\mathrm{rel}}^{*}(p)$ \\
\hline 6 & 1 \\
7 & 0.935127 \\
8 & 0.987060 \\
9 & 0.945933 \\
10 & 0.973952 \\
\hline
\end{tabular}

\begin{tabular}{cc}
\hline$p$ & $H_{\text {rel }}^{*}(p)$ \\
\hline 11 & 0.953759 \\
12 & 0.969485 \\
13 & 0.959646 \\
14 & 0.968378 \\
15 & 0.964221 \\
\hline
\end{tabular}

\begin{tabular}{cc}
\hline$p$ & $H_{\mathrm{rel}}^{*}(p)$ \\
\hline 16 & 0.968695 \\
17 & 0.967874 \\
18 & 0.969629 \\
19 & 0.970855 \\
20 & 0.970814 \\
\hline
\end{tabular}

\section{Main Results}

Let us summarize the conclusions of the previous analysis:

Theorem 6.1. If the partition $\alpha=x_{0}<x_{1}<\ldots<x_{M}=\beta$ of the domain $\Omega=(\alpha, \beta)$ satisfies the condition

$$
\frac{x_{i}-x_{i-1}}{\beta-\alpha} \leq H_{\mathrm{rel}}^{*}\left(p_{i}\right) \quad \text { for all } i=1,2, \ldots, M,
$$

where $p_{i} \geq 1$ is the polynomial degree assigned to the element $K_{i}=\left[x_{i-1}, x_{i}\right]$, and $H_{\mathrm{rel}}^{*}\left(p_{i}\right)$ is defined by (5.12), then the problem (2.2) satisfies the discrete maximum principle (i.e., $u_{h p} \geq 0$ in $\Omega$ for arbitrary $f \in L^{2}(\Omega)$ which is nonnegative a.e. in $\Omega$.

Proof. Let $K_{i}$ be any element. By (5.2), condition (6.1), and Theorem 5.3 it holds that

$$
\left.G_{h p}(x, z)\right|_{K_{i}^{2}}=G_{h p}(\xi, \eta) \geq 0 \quad \text { for all }[x, z] \in K_{i}^{2} .
$$

Thus, $G_{h p}(x, z) \geq 0$ in $\bigcup_{i=1}^{M} K_{i}^{2}$. Lemma 4.3 implies that $G_{h p}(x, z) \geq 0$ also in $\Omega^{2} \backslash \bigcup_{i=1}^{M} K_{i}^{2}$. Theorem 3.5 finishes the proof.

Table 1indicates that the restriction on the relative element length $\left(x_{i}-x_{i-1}\right) /$ $(\beta-\alpha)$ is strongest in the cubic case where $H_{\text {rel }}^{*}=9 / 10$. Moreover, Figure 3 shows a steadily growing trend in $H_{\text {rel }}^{*}$ for $p \geq 50$. These observations motivate the following conjecture:

Conjecture 6.2. If the partition $\alpha=x_{0}<x_{1}<\ldots<x_{M}=\beta$ of the domain $\Omega=(\alpha, \beta)$ satisfies the condition

$$
\frac{x_{i}-x_{i-1}}{\beta-\alpha} \leq \frac{9}{10} \text { for all } i=1,2, \ldots, M,
$$

then problem (2.2) satisfies the discrete maximum principle (i.e., $u_{h p} \geq 0$ in $\Omega$ for arbitrary $f \in L^{2}(\Omega)$ which is nonnegative a.e. in $\Omega$ ).

\section{Possible generalizations}

An analogous technique can be used to study problem (2.1) with mixed DirichletNeumann boundary conditions. Of course, the structure of the stiffness matrix and the structure of the DGF are different, but analysis reveals that the quantity $H_{\text {rel }}^{*}(p)$ plays a central role again. Since $H_{\text {rel }}^{*}(p)$ is nonnegative in this case (at least for $p \leq 100$ ), the DMP for problem (2.1) with mixed boundary conditions is valid with no restricting conditions on the mesh or polynomial degrees of elements. More details can be found in a recent report [19]. 
Generalization of these results to problems with variable coefficients and to higher-dimensional problems, however, will be more involved. In both of these cases, higher-order shape functions are no longer orthogonal, which yields a nontrivial cross term in the expression for the DGF. An analysis of this term will be crucial to achieve any progress in this direction. The goal of the analysis is to infer possibly simple conditions on the mesh and polynomial degrees of elements so that the DMP is valid. To achieve this goal, new techniques for the analysis of the DGF have to be developed.

The negative result from [7] does not imply that generalizations to $2 \mathrm{D}$ are impossible. This paper dealt with a stronger version of the DMP which required the maximum principle to be valid in all subdomains. Basically, the paper showed that the DMP for higher-order elements was not valid on vertex patches (patches of elements surrounding mesh vertices). It seems that vertex patches simply are too coarse for the DMP to be valid.

Another possibility would be to employ an idea from [1] to treat a class of $1 \mathrm{D}$ problems with a variable coefficient

$$
-\left(\varrho(x) u^{\prime}\right)^{\prime}=f, \quad u(\alpha)=u(\beta)=0 .
$$

The idea would be to define new vertex functions to be piecewise-harmonic, such that each $\phi_{i}, i=1,2, \ldots, M-1$, solves

$$
\begin{array}{rlrr}
-\left(\varrho(x) \phi_{i}^{\prime}\right)^{\prime}=0 & \text { on }\left(x_{i-1}, x_{i}\right), & u\left(x_{i-1}\right)=0, & u\left(x_{i}\right)=1, \\
-\left(\varrho(x) \phi_{i}^{\prime}\right)^{\prime}=0 & \text { on }\left(x_{i}, x_{i+1}\right), & u\left(x_{i}\right)=1, & u\left(x_{i+1}\right)=0 .
\end{array}
$$

Such vertex functions, interestingly, would be orthogonal to bubble functions. However, the definition of the corresponding bubble functions and formulation of the condition for the DMP to be valid need further research.

\section{APPENDIX}

The Lobatto shape functions are defined by

$$
l_{j}(\xi)=\sqrt{\frac{2 j-1}{2}} \int_{-1}^{\xi} P_{j-1}(x) \mathrm{d} x, \quad j=2,3, \ldots
$$

where $P_{j}(x)=\mathrm{d}^{j} / \mathrm{d} x^{j}\left(x^{2}-1\right)^{j} /\left(2^{j} j !\right)$ stands for the $j$ th-degree Legendre polynomial. The kernels are defined by $\kappa_{j}(\xi)=l_{j}(\xi) /\left(l_{0}(\xi) l_{1}(\xi)\right)$, where $l_{0}(\xi)=(1-\xi) / 2$, $l_{1}(\xi)=(1+\xi) / 2$, and $\xi \in[-1,1]$. These kernels can be generated by the recurrence

$$
\kappa_{j+2}(\xi)=\frac{\sqrt{2 j+1} \sqrt{2 j+3}}{j+2} \xi \kappa_{j+1}(\xi)-\frac{j-1}{j+2} \sqrt{\frac{2 j+3}{2 j-1}} \kappa_{j}(\xi), \quad j=2,3, \ldots
$$

\footnotetext{
${ }^{2}$ We thank an anonymous referee for pointing this out.
} 
For reference, we list several kernel functions $\kappa_{i}$ (see, e.g., Section 3.1 in [15] or Section 1.2 in [13]):

$$
\begin{aligned}
\kappa_{2}(\xi) & =-\sqrt{6} \\
\kappa_{3}(\xi) & =-\sqrt{10} \xi \\
\kappa_{4}(\xi) & =-\frac{1}{4} \sqrt{14}\left(5 \xi^{2}-1\right) \\
\kappa_{5}(\xi) & =-\frac{3}{4} \sqrt{2}\left(7 \xi^{2}-3\right) \xi \\
\kappa_{6}(\xi) & =-\frac{1}{8} \sqrt{22}\left(21 \xi^{4}-14 \xi^{2}+1\right) \\
\kappa_{7}(\xi) & =-\frac{1}{8} \sqrt{26}\left(33 \xi^{4}-30 \xi^{2}+5\right) \xi \\
\kappa_{8}(\xi) & =-\frac{1}{64} \sqrt{30}\left(429 \xi^{6}-495 \xi^{4}+135 \xi^{2}-5\right), \\
\kappa_{9}(\xi) & =-\frac{1}{64} \sqrt{34}\left(715 \xi^{6}-1001 \xi^{4}+385 \xi^{2}-35\right) \xi \\
\kappa_{10}(\xi) & =-\frac{1}{128} \sqrt{38}\left(2431 \xi^{8}-4004 \xi^{6}+2002 \xi^{4}-308 \xi^{2}+7\right) .
\end{aligned}
$$

\section{ACKNOWLEDGMENTS}

The first author has been supported by the Grant Agency of the Czech Republic, project No. 201/04/P021 and by the Academy of Sciences of the Czech Republic, Institutional Research Plan No. AV0Z10190503. The second author has been supported in part by the U.S. Department of Defense under the Grant No. 05PR07548-00, by the NSF Grant No. DMS-0532645, and by the Grant Agency of the Czech Republic, project No. 102-05-0629. This support is gratefully acknowledged.

\section{REFERENCES}

1. I. Babuška, G. Caloz, J. Osborn, Special finite element methods for a class of second order elliptic problems with rough coefficients, SIAM J. Numer. Anal., 31 (1994), pp. 945-981. MR.1286212 (95g:65146)

2. E. Burman, A. Ern, Discrete maximum principle for Galerkin approximations of the Laplace operator on arbitrary meshes, C. R. Math. Acad. Sci. Paris 338 (2004), 641-646. MR2056474

3. P.G. Ciarlet, Discrete maximum principle for finite difference operators, Aequationes Math. 4 (1970), 338-352. MR0292317 (45:1404)

4. P.G. Ciarlet, P.A. Raviart, Maximum principle and uniform convergence for the finite element method, Computer Methods Appl. Mech. Engrg. 2 (1973), 17-31. MR0375802 (51:11992)

5. A. Drăgănescu, T.F. Dupont, L.R. Scott, Failure of the discrete maximum principle for an elliptic finite element problem, Math. Comp. 74 (2005), 1-23 (electronic). MR2085400 (2005f:65148)

6. M. Fiedler, Special matrices and their applications in numerical mathematics, Martinus Nijhoff Publishers, Dordrecht, 1986. MR.1105955 (92b:15003)

7. W. Höhn, H.D. Mittelmann, Some remarks on the discrete maximum principle for finite elements of higher-order, Computing 27 (1981), 145-154. MR632125 (83a:65109)

8. A. Jüngel, A. Unterreiter, Discrete minimum and maximum principles for finite element approximations of non-monotone elliptic equations, Numer. Math. 99 (2005), 485-508. MR2117736 (2005m:65269)

9. J. Karátson, S. Korotov, Discrete maximum principles for finite element solutions of nonlinear elliptic problems with mixed boundary conditions, Numer. Math. 99 (2005), 669-698. MR 2121074 (2005k:65253) 
10. S. Korotov, M. Křížek, P. Neittaanmäki, Weakened acute type condition for tetrahedral triangulations and the discrete maximum principle, Math. Comp. 70 (2000), 107-119. MR 1803125 (2001i:65126)

11. A.H. Schatz, A weak discrete maximum principle and stability of the finite element method in $L_{\infty}$ on plane polygonal domains. I, Math. Comp. 34 (1980), 77-91. MR551291|(81e:65063)

12. P. Šlín, Partial differential equations and the finite element method, J. Wiley \& Sons, 2005. MR 2180081 (2006f:35004)

13. P. Solín, K. Segeth, I. Doležel, Higher-order finite element methods, Chapman \& Hall/CRC Press, Boca Raton, 2003.

14. P. Šolín, T. Vejchodský, A weak discrete maximum principle for $h p$-FEM, J. Comput. Appl. Math., 2006 (to appear).

15. B. Szabó, I. Babuška, Finite element analysis, John Wiley \& Sons, New York, 1991. MR 1164869 (93f:73001)

16. R.S. Varga, Matrix iterative analysis, Englewood Cliffs, New Jersey, Prentice-Hall, 1962. MR 0158502 (28:1725)

17. T. Vejchodský, On the nonnegativity conservation in semidiscrete parabolic problems. In: M. Křížek, P. Neittaanmäki, R. Glowinski, S. Korotov (Eds.), Conjugate gradients algorithms and finite element methods, Berlin, Springer-Verlag, 2004, pp. 197-210. MR2082563 (2005i:65135)

18. T. Vejchodský, Method of lines and conservation of nonnegativity. In: Proc. of the European Congress on Computational Methods in Applied Sciences and Engineering (ECCOMAS 2004), Jyväskylä, Finland, 2004.

19. T. Vejchodský, P. Šolín, Discrete Maximum Principle for Mixed Boundary Conditions in 1D, Research Report No. 2006-09, Department of Math. Sciences, University of Texas at El Paso, July 2006.

20. J. Xu, L. Zikatanov, A monotone finite element scheme for convection-diffusion equations, Math. Comp. 68 (1999), 1429-1446. MR1654022 (99m:65225)

21. E.G. Yanik, Sufficient conditions for a discrete maximum principle for high-order collocation methods, Comput. Math. Appl. 17 (1989), 1431-1434. MR999250 (90c:65106)

Mathematical Institute, Academy of Sciences, Žitná 25, Praha 1, CZ-115 67, Czech REPUBLIC

E-mail address: vejchod@math.cas.cz

Institute of Thermomechanics, Academy of Sciences, Dolejškova 5, Praha 8, CZ182 00, Czech Republic

Current address: Department of Mathematical Sciences, University of Texas at El Paso, El Paso, Texas 79968-0514

E-mail address: solin@utep.edu 